\title{
The Importance of Cell-Cell Interaction Dynamics in Bottom-Up Tissue Engineering: Concepts of Colloidal Self-Assembly in the Fabrication of Multicellular Architectures
}

\author{
Marc Mueller, ${ }^{\dagger}$ Samaneh Rasoulinejad, ${ }^{\dagger}$ Sukant Garg, ${ }^{\dagger}$ and Seraphine V. Wegner*, ${ }^{*, \ddagger}$ \\ ${ }^{\dagger}$ Max Planck Institute for Polymer Research, 55128 Mainz, Germany \\ ${ }^{\ddagger}$ Institute of Physiological Chemistry and Pathobiochemistry, University of Münster, 48149 Münster, Germany \\ Supporting Information
}

ABSTRACT: Building tissue from cells as the basic building block based on principles of self-assembly is a challenging and promising approach. Understanding how far principles of self-assembly and self-sorting known for colloidal particles apply to cells remains unanswered. In this study, we demonstrate that not just controlling the cell-cell interactions but also their dynamics is a crucial factor that determines the formed multicellular structure, using photoswitchable interactions between cells that are activated with blue light and reverse in the dark. Tuning dynamics of the cell-cell interactions by pulsed light activation results in multicellular architectures with different sizes and shapes. When the interactions between cells are dynamic, compact and round multicellular clusters under thermodynamic control form, while otherwise branched and loose aggregates under kinetic control assemble. These structures parallel what is known for colloidal assemblies under reaction- and diffusion-limited cluster

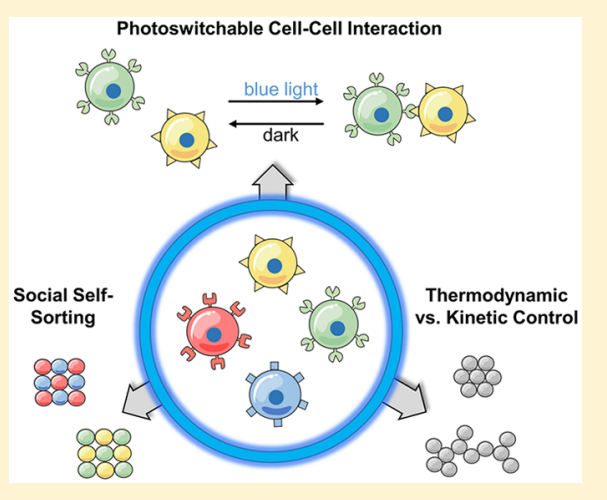
aggregation, respectively. Similarly, dynamic interactions between cells are essential for cells to self-sort into distinct groups. Using four different cell types, which expressed two orthogonal cell-cell interaction pairs, the cells sorted into two separate assemblies. Bringing concepts of colloidal self-assembly to bottom-up tissue engineering provides a new theoretical framework and will help in the design of more predictable tissue-like structures.

KEYWORDS: Bottom-up tissue engineering, optogenetics, cell-cell interactions, self-assembly, self-sorting, photoswitchable

$\mathrm{T}$ he bottom-up assembly of a spatially ordered tissue made from cellular building blocks based on the principles of self-assembly is a highly promising and powerful approach to tissue engineering and an extreme synthetic and biological challenge at the same time. ${ }^{1}$ Building multicellular structures requires more than simply putting together a solution with the right composition of cells; it requires specific interactions between the cells and spatial organization of these building blocks into hierarchical structures, which determines how cells work together as a tissue. ${ }^{2,3}$ The bottom-up approach to tissue assembly parallels observations seen during tissue formation in biology, where no template or scaffold is needed and cell-cell interactions are a major driving force that determines their organization. ${ }^{4}$ Remarkably, even dissociated cells from different tissues are able to self-aggregate and self-sort again into multicellular structures that resemble their tissues of origin., Moreover, increasing possibilities in organoid ${ }^{7}$ and stem cell culture $^{5,6}$ as well as programmable multicellular structures with synthetic cell to cell signaling ${ }^{7}$ speaks for the massive potential of the living cells to self-organize into complex functional architectures and controlling them using synthetic biology.

Going forward, it is indispensable to understand how cells as the basic building blocks of tissue self-assembly. This requires controlling the interactions between cellular building blocks and understanding to what extent the principles of selfassembly and self-sorting known for nonliving colloidal particles apply to cells. ${ }^{9}$ Such insight would allow us to build up multicellular architectures with predictable and programmable organization, understand the limits of multicellular structures that can be generated solely based on self-assembly and where further biological signals are required. ${ }^{1}$ As model building blocks for materials, colloids provide a valuable framework for the self-assembly of micron-sized objects such as the cells. ${ }^{10}$ For colloidal systems, the interactions between colloids are the major driving force behind self-assembly, and different architectures can self-assemble depending on the kinetic and thermodynamic parameters for the interactions between the colloids. ${ }^{11}$ While compact and spherical structures at the thermodynamic equilibrium form under reaction-limited cluster aggregation (RLCA), loose and ramified assemblies in kinetically trapped states form under diffusion-limited cluster aggregation (DLCA). ${ }^{12-14}$ This puts forward the importance of not just controlling the interactions between the colloidal/ cellular building blocks but also their dynamics. Cell-cell

Received: October 9, 2019

Revised: November 19, 2019

Published: November 21, 2019 
interactions have been controlled by modifying the surfaces of cells with complementary DNA strands, ${ }^{15}$ biotin-streptavidin, ${ }^{16}$ clickable functional groups, ${ }^{17,18}$ supramolecular interaction partners, ${ }^{18,19}$ and the photoswitchable protein pair CRY2/CIBN. ${ }^{20}$ Yet, except for the last two methods, these interactions are not reversible and provide no control over the dynamics of the cell-cell interactions. Moreover, the important role of the cell-cell interaction dynamics for the final multicellular structure has not been considered in any of these studies. This is in contrast to native cadherin based cellcell adhesions, which have fast exchange rates and form thermodynamically controlled multicellular structures. ${ }^{21}$ Therefore, the question of what kind of tissue structures can be generated by employing only the principles of self-assembly and controlling cell-cell interactions to achieve diffusion or reaction-limited assembly remains unanswered.

Another concept where the principles of colloids and cells connect to one another is their self-sorting/sorting-out behavior in multicomponent mixtures. ${ }^{22,23}$ Observations in in vivo and in vitro multicellular systems led to the differential adhesion hypothesis, which postulates that, if two populations of cells are mixed, the cells sort-out to reach a final organization that approaches a state with a minimal internal free energy and maximum total cell-cell interactions. ${ }^{24}$ Such self-sorting under thermodynamic control is only possible provided that the cell-cell interactions are dynamic, and this criterion is indeed satisfied for native cadherin-based cell-cell interactions. ${ }^{21}$ Consequently, in mixtures of dissociated cells that express different types or levels of cadherins, the cells sortout to form self-isolated, enveloped, and intermixed multicellular structures depending on their preference to bind to cells of the same or opposite type. ${ }^{21,25}$ Yet, also other mechanisms of self-sorting that rely on local cell signaling or contractile properties of cells have also been proposed and add to the complexity of multicellular systems. ${ }^{26}$ Similarly, multicolloidal mixtures self-sort into families of colloids based on multiple molecularly orthogonal homophilic and heterophilic interactions between different types of colloids. $^{27-29}$ For example, mixtures of four distinct colloids self-sort into two families of colloidal aggregates using two orthogonal heterodimerization pairs by virtue of a behavior named social self-sorting. ${ }^{27,29}$

Here, we employ concepts known from colloidal selfassembly and explore how far these can be used in the context of multicellular structures (Figure 1a). For this purpose, we establish different photoswitchable cell-cell interactions, which can be triggered under blue light illumination and turned off in the dark with different protein-protein interaction dynamics and dark reversion rates. Controlling the cell-cell interaction with light comes with the unique advantage of high spatiotemporal resolution and turning on the cell-cell adhesions remotely using low-intensity biocompatible light without interfering with other cellular processes. Most importantly, regulation with light allows tuning cell-cell interactions dynamically by using pulses of light. These unique features enabled us to investigate how the thermodynamics and kinetics of the interactions between the cellular building blocks impact the multicellular assemblies and achieve selfassembly under kinetic and thermodynamic control, as has been described for colloidal systems. Moreover, combining different orthogonal cell-cell interactions allowed us to not only self-assemble but also self-sort mixtures of four different cell types into separate preferential assemblies.
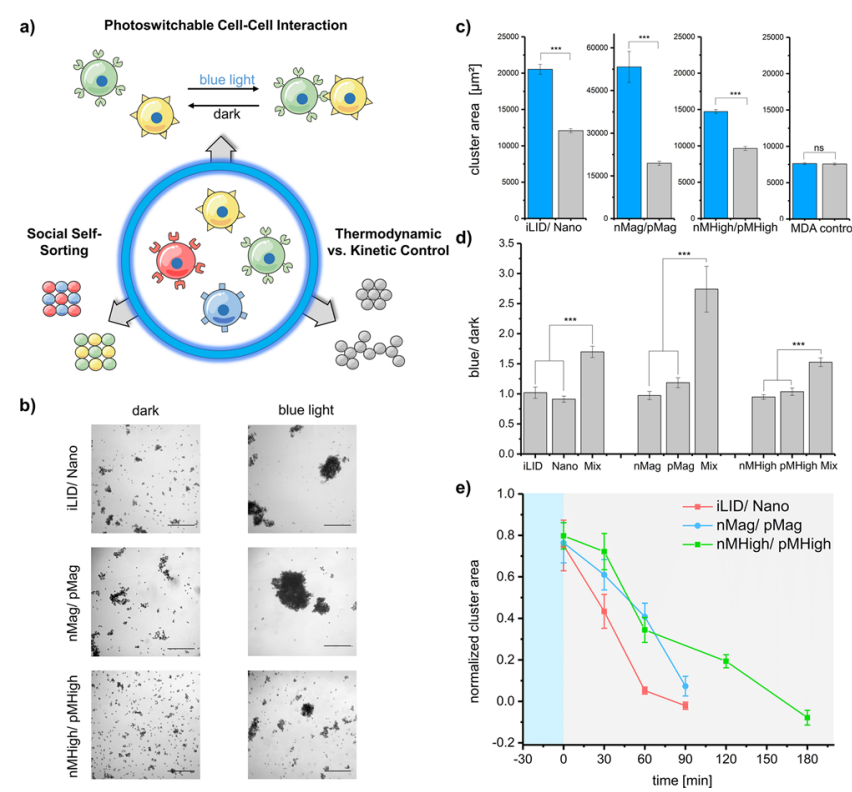

Figure 1. Photoswitchable cell-cell interactions with different dynamics. (a) Schematic representation of cells expressing different photoswitchable proteins at their surface form cell-cell interactions under blue light and dissociate in the dark. The final structure of the multicellular assemblies can be kinetically or thermodynamically controlled, depending on the cell-cell interaction dynamics. If four different cell types, expressing two orthogonal heterophilic interaction pairs, are mixed, they can self-sort into two separate assemblies, known as social self-sorting. (b) Bright-field images of iLID-/NanoMDA, nMag-/pMag-MDA, and nMagHigh-/pMagHigh-MDA cells in the dark and under blue light after $30 \mathrm{~min}$ at $20 \mathrm{rpm}$. Scale bars are $500 \mu \mathrm{m}$. (c) Quantification of the cell aggregation. (d) Ratio of the cluster sizes under blue light and in the dark for mono and mixed cultures. A ratio of 1 shows no light-dependent cell aggregation. (e) Reversibility of the cell-cell interactions in the dark after $30 \mathrm{~min}$ preillumination with blue light. The cluster area was normalized to control samples kept under blue light and in the dark for the whole duration of the experiment (Supporting Information, Figure S5). At least 25 images with a total area of $1 \mathrm{~cm}^{2}$ were analyzed in each sample, each done in biological duplicates with 3 technical replicates. Error bars are the standard error of the mean cluster area, $p$-value < 0.001 represented as $* * *$.

We first focused on establishing different photoswitchable cell-cell interactions with different binding strengths, proteinprotein interaction dynamics, and reversion kinetics in the dark. For this purpose, we expressed different light-dependent protein-protein interaction partners as synthetic adhesion receptors on the surfaces of the breast cancer cell line MDAMB-231, which do not form strong native cell-cell adhesions. $^{30}$ In particular, we used three protein pairs that specifically heterodimerize with each other under blue light $(450 \mathrm{~nm})$ and dissociate from each other in the dark, named iLID and Nano (dark reversion rate $3.5 \times 10^{-2} \mathrm{~s}^{-1}, t_{1 / 2}=20$ $\mathrm{s}){ }^{31} \mathrm{nMag}$ and $\mathrm{pMag}$ (dark reversion rate $1.1 \times 10^{-4} \mathrm{~s}^{-1}, t_{1 / 2}=$ $1.8 \mathrm{~h}$ ), and nMagHigh and pMagHigh (dark reversion rate 4.1 $\left.\times 10^{-5} \mathrm{~s}^{-1}, t_{1 / 2}=4.7 \mathrm{~h}\right){ }^{32}$ These proteins were chosen due to the large range of dark reversion times they cover, their different protein-protein interaction dynamics, the tunability of their interactions with few point mutations (e.g., nMag/ pMag vs nMagHigh/pMagHigh), and their similar size, which presumably will lead to a similar expression level on the cell surface. To express these proteins on the cell surface each of the genes coding for them were cloned into a pDisplay vector, 
which, once the protein is expressed, guides it to the cell membrane with an $\mathrm{N}$-terminal murine $\operatorname{Ig} \kappa$-chain leader sequence and anchors it at the cell membrane with a Cterminal platelet-derived growth factor receptor (PDGFR) transmembrane domain (Supporting Information, Figure S1). Plasmids coding for different proteins were individually transfected into MDA-MB-231 cells, and stable monoclonal cell lines expressing these proteins at their surfaces were generated. The cell lines were named after the protein expressed at their surface; e.g., iLID expressing cells were named iLID-MDA. For each photoswitchable protein, a single clone with high protein expression was selected, and the expression of each protein on the cell surface was confirmed by flow cytometry and immunostaining (Supporting Information, Figures S2 and S3). On the surfaces of the cells $6 \times 10^{3}$ to $5 \times$ $10^{4}$ photoswitchable proteins per cell were expressed as measured by quantitative flow cytometry, showing comparable expression levels of the different photoswitchable proteins on the cell surface (Supporting Information, Table S2). In this study, we used MDA-MB-231 cells to demonstrate the concept, yet these genetically encoded photoswitchable proteins could be transfected and used to mediate cell-cell interactions between other cell types too.

To see if the photoswitchable proteins can mediate lighttriggered cell-cell interactions, cells expressing complementary interaction partners (iLID-MDA and Nano-MDA, nMag-MDA and pMag-MDA, nMagHigh-MDA, and pMagHigh-MDA) were incubated in suspension in the dark and under blue light illumination for $30 \mathrm{~min}$. The mixed cultures of two complementary cell types aggregated significantly under blue light but remained scattered in the dark, as observed in brightfield images (Figure $1 \mathrm{~b}$ ). To quantify the cell aggregation, large areas of the samples were scanned $\left(1-2.56 \mathrm{~cm}^{2}\right.$ per sample containing about 25000 cells $/ \mathrm{cm}^{2}$ ), and cell aggregates with a two-dimensional projected area of larger than $5000 \mu \mathrm{m}^{2}$, i.e., containing at least 20 cells, were detected as clusters using automated image analysis. In each of the three cocultures, blue light resulted in the assembly of multicellular structures with a significantly higher mean cluster area than in the dark (Figure 1c). Moreover, the blue-light-dependent cell aggregation was due to the specific heterophilic interactions between the different photoswitchable proteins, and homophilic interactions did not contribute to the aggregation, as in none of the monocultures containing just one cell type blue light illumination increased the cell aggregation (Figure $1 \mathrm{~d}$, Supporting Information, Figure S4).

One reason the protein pairs, iLID/Nano, nMag/pMag, and $\mathrm{nMagHigh/pMagHigh,} \mathrm{were} \mathrm{selected} \mathrm{is} \mathrm{due} \mathrm{to} \mathrm{their} \mathrm{different}$ reversion kinetics in the dark. ${ }^{31,32}$ The reversibility of cell-cell interactions is a key feature of cell-cell adhesions in biology and indispensable for the self-sorting following the differential adhesion hypothesis. When cocultures of cells expressing complementary interaction partners were preaggregated for 30 min under blue light illumination and then placed in the dark, all three aggregate types dissociated, yet with different time dependences (Figure 1e, Supporting Information, Figure S5). The aggregates in iLID-/Nano-MDA cocultures disassembled the fastest within $60 \mathrm{~min}$, aggregates in $\mathrm{nMag}-/ \mathrm{pMag}-\mathrm{MDA}$ cocultures disassembled within $90 \mathrm{~min}$, and aggregates in nMagHigh-/pMagHigh-MDA cocultures disassembled the slowest over $180 \mathrm{~min}$. This trend corresponds to reversion time at the molecular level, which is iLID/Nano < nMag/ pMag < nMagHigh/pMagHigh. ${ }^{31,32}$ The disparity in the absolute values for the reversion for the cell-cell interactions to the protein level could potentially be due to multivalent protein-protein interactions between cells, processes that are coupled to the cell-cell interactions beyond the photoswitching at the molecular level and the display of the proteins on the extracellular cell surface.

The second striking difference between different photoswitchable protein pairs was the morphology of the multicellular aggregates formed (Figures $1 \mathrm{~b}$ and $2 \mathrm{a}$ ). iLID-/Nano-

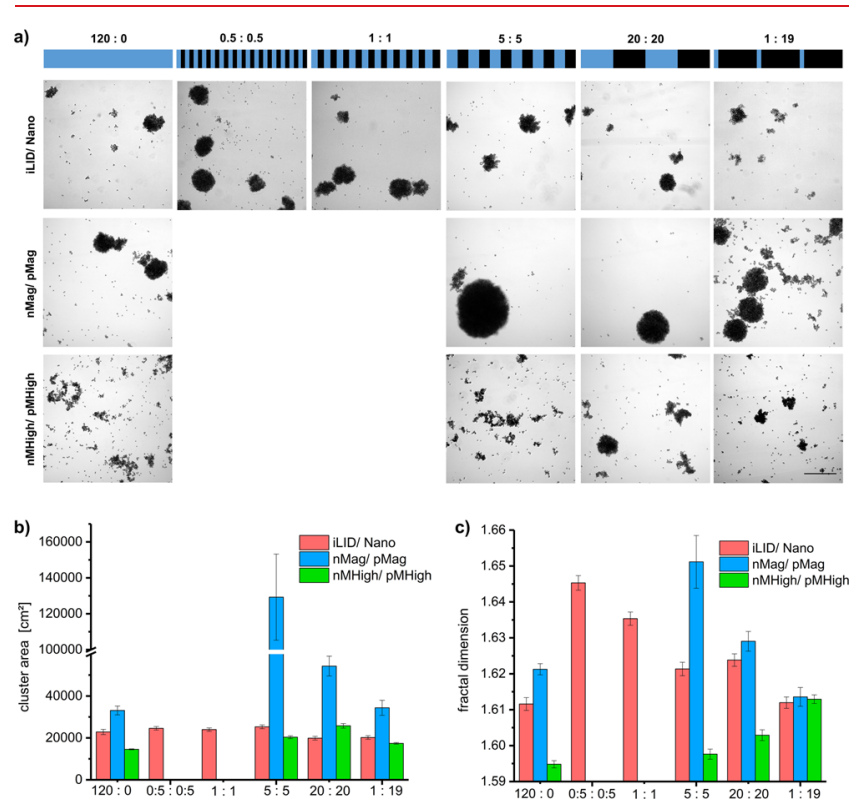

Figure 2. Multicellular assemblies under kinetic and thermodynamic control. (a) Bright-field images of iLID-/Nano-MDA, nMag-/pMagMDA and nMagHigh-/pMagHigh-MDA cells with different blue light pulsing sequences (120 min continues blue light, $0.5 \mathrm{~min}$ on $/ 0.5 \mathrm{~min}$ off (only for iLID-/Nano-MDA), $1 \mathrm{~min}$ on/ $1 \mathrm{~min}$ off (only for iLID-/ Nano-MDA), $5 \mathrm{~min}$ on $/ 5 \mathrm{~min}$ off, $20 \mathrm{~min}$ on $/ 20 \mathrm{~min}$ off, $1 \mathrm{~min}$ on/ $19 \mathrm{~min}$ off) after $2 \mathrm{~h}$. Scale bars are $500 \mu \mathrm{m}$. Quantification of (b) the mean cluster area and (c) fractal dimension with different illumination protocols. Here, 64 images with a total area of $2.56 \mathrm{~cm}^{2}$ were analyzed for each sample, each done in biological duplicates with 3 technical replicates. Error bars are the standard error of the mean cluster area, $p$-value $<0.001$ represented as $* * *$.

MDA cocultures and nMag-/pMag-MDA cocultures formed compact aggregates with smooth edges after $2 \mathrm{~h}$ under blue light. On the other hand, in nMagHigh-/pMagHigh-MDA cocultures under the same conditions loose and ramified aggregates with irregular shapes formed. Furthermore, iLID-/ Nano-MDA and nMag-/pMag-MDA aggregates were also larger than nMagHigh-MDA/pMagHigh-MDA aggregates. These observations suggest that the dynamics of the protein-protein interactions and their interaction strengths play an important role in the self-assembly of multicellular structures. While iLID-/Nano-MDA and nMag-/pMag-MDA aggregates exemplify the RLCA dominated by thermodynamic control, aggregates of nMagHigh-/pMagHigh-MDA cells are examples of the DLCA and are mostly under kinetic control. This data also mirrors the reaction- and diffusion-limited cluster aggregation observed in colloidal polystyrene particles coated with iLID and Nano or nMagHigh and pMagHigh, respectively. ${ }^{29}$ These observations directly correlate with the stronger protein-protein interaction between nMagHigh/ pMagHigh and slower on/off rates compared to the weaker 
and more dynamics protein-protein interaction between nMag/pMag and iLID/Nano. ${ }^{33}$

Next, we wanted to explore whether we could shift the selfassembled multicellular architectures from kinetically to thermodynamically controlled structures by altering the strength and dynamics of the cell-cell interactions. The photoswitchable cell-cell interactions provide a unique opportunity to address this question as protein-protein interaction strength and dynamics can be tuned using pulses of light. ${ }^{34,35}$ For this purpose, we incubated different cocultures under blue light illumination with varying on and off times for a total of $2 \mathrm{~h}$ (continuous $120 \mathrm{~min}$ on, $0.5 \mathrm{~min}$ on $/ 0.5 \mathrm{~min}$ off (only for iLID-/Nano-MDA), $1 \mathrm{~min}$ on/ $1 \mathrm{~min}$ off (only for iLID-/Nano-MDA), $5 \mathrm{~min}$ on/5 min off, $20 \mathrm{~min}$ on $/ 20 \mathrm{~min}$ off, $1 \mathrm{~min}$ on $/ 19 \mathrm{~min}$ off) (Figure $2 \mathrm{a}$ ). We observed that different multicellular aggregates formed depending on the illumination frequency. Outstandingly, less total illumination but in pulses lead to an increase in cell aggregation for iLID-/ Nano-MDA cells (0.5 min on/0.5 min off), nMag-/pMagMDA cells ( $5 \mathrm{~min}$ on $/ 5 \mathrm{~min}$ off), as well as nMagHigh-/ pMagHigh-MDA cells (5 min on/5 min off and $20 \mathrm{~min}$ on $/ 20$ min off) as also evident by the increase in the mean cluster area (Figure 2a,b, Supporting Information, Figure S6). This shows that pulsed illumination can lead to increased aggregation if the cell-cell interactions partially revert, and cells can reposition themselves when the light is off such that, upon reactivation with blue light, cells can optimize their position and increase interactions with their neighbors. For this reason, slower pulsing (20 $\mathrm{min}$ on/20 $\mathrm{min}$ off) enhances aggregation for nMagHigh-/pMagHigh-MDA cells with slower dark reversion and faster pulsing for iLID-/Nano-MDA and nMag/pMag cells (0.5 $\mathrm{min}$ on/0.5 $\mathrm{min}$ off, $5 \mathrm{~min}$ on $/ 5 \mathrm{~min}$ off, respectively) with faster dark reversion. On the other hand, a longer off time (20 $\mathrm{min}$ on $/ 20 \mathrm{~min}$ off) or less photoactivation $(1 \mathrm{~min}$ on/19 min off) leads to a decrease in aggregation in all three photoswitchable cell-cell interaction pairs. This trend was best observed with nMag-/pMag-MDA cell aggregation, which increased with $5 \mathrm{~min}$ on $/ 5 \mathrm{~min}$ off pulsing compared to continuous illumination, but decreased with lower pulsing frequency ( $20 \mathrm{~min}$ on $/ 20 \mathrm{~min}$ off $)$, although the total light dose was the same and even further if the photoactivation was decreased ( $1 \mathrm{~min}$ on/19 min off). Thus, if the reversion of the cell-cell interactions in the dark is extensive or the reactivation with blue light is not sufficient, aggregates disassemble, and when the dark reversion time of the photoswitchable protein is shorter, this disassembly is more pronounced. Taken together, this data shows that not only the cell-cell interaction strength but also their dynamics here modulated with pulsed illumination are critical for the self-assembly of multicellular structures.

The second aspect that is closely related to the cell-cell interaction dynamics is the morphology of the multicellular assemblies, which vary from loose and ramified to compact and spherical going from DLCA to RLCA. ${ }^{14}$ As observed above (Figure 2), aggregation increases when the cell-cell interaction is only partially reversed with pulsing, so that the cells could transiently reposition and strengthen their contact with neighboring cells, which represents a shift from kinetically to thermodynamically controlled structures. To rationalize and quantify the relationship between the morphology of the cluster and interaction dynamics, we determined the fractal dimension of the two-dimensional contours of these multicellular aggregates as a measure of cluster shape complexity and $\operatorname{size}^{36}$ (Figure 2c, Supporting Information, Figure S7). For comparison in colloidal systems, the fractal dimension increases from 1.46 for DLCA to 1.55 for RLCA for twodimensional aggregates. ${ }^{37}$ For the cellular assemblies, we observed a significant range of fractal dimensions from 1.595 for nMagHigh-/pMagHigh-MDA cells under constant blue light dominated by DLCA to 1.651 for nMag-/pMag-MDA cells with 5 min pluses of blue light dominated by RLCA (Figure 2c). Under constant activation, the fractal dimension was higher for assemblies based on more dynamic proteinprotein interactions, (iLID/Nano and nMag/pMag), achieving thermodynamically driven structures. On the contrary, nMagHigh-/pMagHigh-MDA cells formed stronger and less dynamic protein-protein interactions, leading to kinetically trapped structures with a lower fractal dimension. For comparison, we analyzed the fractal dimension of previously reported colloidal aggregates between iLID and Nano as well as nMagHigh and pMagHigh coated $2 \mu$ m polystyrene beads, ${ }^{29}$ and we observed similar trends as with the cellular aggregates. The iLID/Nano-mediated colloidal aggregates had a higher fractal dimension than the nMagHigh/pMagHigh-mediated colloidal aggregates (1.578 vs 1.562 ) (Supporting Information, Table S3). This analysis further confirms the parallels in the colloidal and cellular aggregates.

Pulsed photoactivation increases the dynamics of the cellcell interactions and gives the cells an opportunity to rearrange and form a thermodynamically more stable structure, shifting the assembly from DLCA to RLCA as observed for all cell types. As shown in Figure 2c, nMagHigh-/pMagHigh-MDA cells under constant blue light formed branched clusters with a low fractal dimension (1.595), which increased up to 1.61 as the time in the dark increased and the photoactivation time decreased. Likewise, for nMag-/pMag-MDA and iLID-/NanoMDA assemblies, the fractal dimension increased when $5 \mathrm{~min}$ on $/ 5 \mathrm{~min}$ off and $0.5 \mathrm{~min}$ on/ $0.5 \mathrm{~min}$ off pulsing was used, respectively, compared to continuous blue light illumination. Beyond $5 \mathrm{~min}$ on $/ 5 \mathrm{~min}$ off pulsing for nMag-/pMag-MDA and $0.5 \mathrm{~min}$ on/0.5 min off pulsing for iLID-/Nano-MDA cells, both the cluster size and fractal dimension reduced, suggesting excessive disassembly with an increase in reversion time in the dark. Moreover, the pulsing frequency required to achieve more thermodynamically controlled assemblies, i.e., RLCA, is closely connected to the dark reversion kinetics of the photoswitchable proteins. While $0.5 \mathrm{~min}$ on/ $0.5 \mathrm{~min}$ off was best for the iLID/Nano pair with the faster dark reversion kinetics, $5 \mathrm{~min}$ on $/ 5 \mathrm{~min}$ off pulsing was the best for the $\mathrm{nMag} / \mathrm{pMag}$ pair and the nMagHigh/pMagHigh interactions with the slowest dark reversion kinetics required longer dark periods (ca. $20 \mathrm{~min}$ ) and less photoactivation to release the kinetically trapped structures and transform them into more compact assemblies.

Sorting-out/self-sorting is an important mechanism in nature to form multicellular structures out of multiple cell types and organize them in subdomains, as observed during embryogenesis and in vitro reconstitution studies of different tissue types. ${ }^{25}$ Achieving self-sorting in the context of bottomup tissue engineering requires multiple orthogonal cell-cell interaction pairs with different interaction strengths, and each of these must be dynamic enough for cells to maximize the interactions with neighboring cells. If the cell-cell interactions are not dynamic enough, kinetically trapped architectures away from the thermodynamic optimum with no self-sorting form could form. 
To achieve sorting-out and multicellular structures with subdomains, we mixed four different cell types expressing two orthogonal protein pairs at their surface. In particular, we mixed iLID-/Nano-MDA expressing cells (each stained in red) with either nMag-/pMag-MDA or nMagHigh-/pMagHighMDA expressing cells (each stained in green) to check if their orthogonal specificity could result into self-sorting in a mixed culture (Figure 3a). ${ }^{29}$ Cells expressing nMag, pMag,

a)

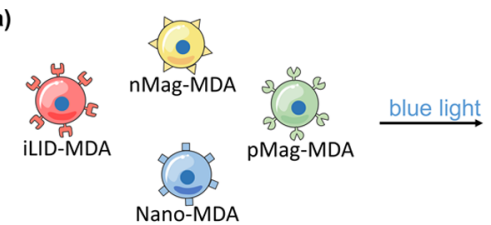

b) iLID-/ Nano-/ nMag-/ pMag-MDA

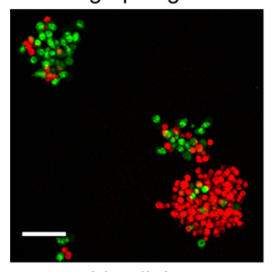

blue light

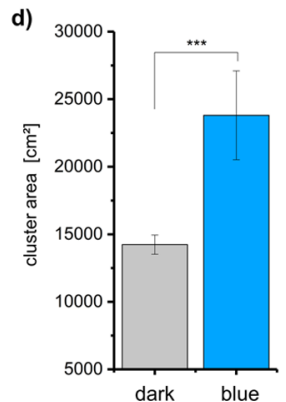

c)

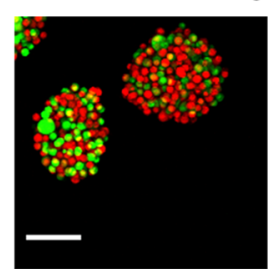

blue light

()

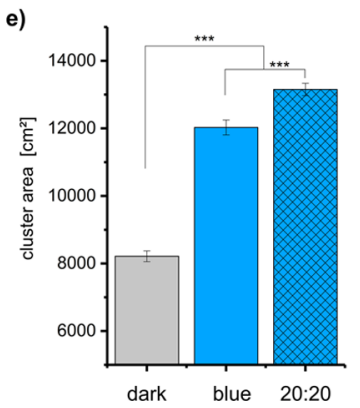

Figure 3. Blue-light-induced social self-sorting in mixtures of four cell types. (a) Schematic overview of social self-sorting of iLID-/NanoMDA cells and nMag-/pMag-MDA cells into separate clusters under blue light. (b) Confocal images of the prestained iLID-/Nano-MDA cells (shown in red) and nMag-/pMag-MDA cells (shown in green) under blue light. (c) Confocal images of prestained iLID-/NanoMDA cells (shown in red) and nMagHigh-/pMagHigh-MDA cells (shown in green) under constant and pulsed blue light (20 min on/20 min off). All scale bars are $100 \mu \mathrm{m}$. Quantification of the mean cluster area for four cell-type mixtures in (d) panels b and (e) c. Each experiment was performed in two biological replicates with technical triplicates. Here, 64 images with a total area of $2.56 \mathrm{~cm}^{2}$ were analyzed in each sample, each done in biological duplicated with 3 technical replicates. Error bars are the standard error of the mean cluster area, $p$-value $<0.001$ represented as $* * *$.

nMagHigh, and pMagHigh were not combined as these proteins bind to one another. ${ }^{32}$ In both of the four-component mixtures, we observed higher light-dependent aggregation under continuous blue light illumination than in the dark overnight (Figure 3b,d,e, Supporting Information, Figure S8), yet the aggregates differed in the organization of the different cell types. In the former mixture, iLID- and Nano-MDA cells (stained in red) clustered separately from the nMag- and pMag-MDA cells (stained in green), showing social sorting of the four cell types (Figure $3 \mathrm{~b}$ ). On the other hand, in the mixture of iLID-, Nano-, nMagHigh-, and pMagHigh-MDA cells, the green- and red-labeled cells were homogeneously intermixed within the same multicellular structure, and the four cell types aggregated together (Figure $3 c$, left). The social selfsorting of the cells was further quantified by analyzing the colocalization of the red and green fluorescent cells using the threshold overlap score (TOS) (Supporting Information, Figure S9). This analysis showed lower colocalization in cocultures of iLID-, Nano- with nMag- and pMag-MDA cells compared to cocultures where nMagHigh- and pMagHighMDA cells were used instead. The fact that self-sorting, specifically social self-sorting, ${ }^{29}$ was observed combining the more dynamic cell-cell interaction pairs, viz., iLID-/NanoMDA and nMag-/pMag-MDA, which favor thermodynamically controlled assemblies and not the nMagHigh/pMagHigh pair, which forms kinetically trapped structures, also demonstrates the importance of dynamics in self-sorting. In an attempt to increase the dynamics between the nMagHigh-/ pMagHigh-MDA cells, $20 \mathrm{~min}$ on $/ 20 \mathrm{~min}$ off pulsing was used to achieve self-sorting within the four-component mixture. The pulsing increased the area of the clusters, and yet did not result in the complete self-sorting, and only domains of green- and red-labeled cells formed within the same aggregate, which was also reflected in an intermediate TOS compared to the selfsorting and nonsorting cocultures (Figure 3c, right, Supporting Information, Figure S9).

In this study, we demonstrate the importance of cell-cell interaction dynamics in the assembly and self-sorting of multicellular structures from cells as building blocks under kinetic or thermodynamic control. Blue-light-triggered cellcell interactions based on different photoswitchable protein interactions (iLID/Nano, nMag/pMag, and nMagHigh/ pMagHigh) with various binding strengths, protein-protein interaction dynamics and dark reversion kinetics provide unique tools for modulating cell-cell interaction dynamics. Using different interaction pairs and the temporal control that light as a stimulus provides, we were able to assemble and tune multicellular structures from branched and ramified to compact and spherical. Moreover, in mixtures with four different cell types, we were able to achieve self-sorting provided that the cell-cell interactions were dynamic enough, as also postulated by the differential adhesion hypothesis. These findings showed that concepts of DLCA and RLCA aggregation as well as of self-sorting that are well-established for colloidal systems can also be applied to the self-assembly of cells into tissue-like architectures. While to date, cell-cell interactions have been controlled using chemical and genetic approaches, and the importance of cell-cell interaction dynamics has not been considered. Most chemical approaches using DNA, clickable groups, and biotin-streptavidin form strong interactions with low exchange rates and are hence expected to result in DLCA, which represents kinetically controlled branched structures. On the other hand, introducing different cadherins to the cell surface, which form highly dynamic protein-protein interactions, results in RLCA with round assemblies under thermodynamic control. In terms of dynamics, the photoswitchable cell-cell interactions based on different photoswitchable proteins offer a wide range of interaction dynamics and strengths, which can be modulated to achieve both kinetically and thermodynamically driven multicellular assemblies. In this respect, bringing basic concepts of colloidal self-assembly to bottom-up tissue engineering will help in the design of more predictable and complex microtissue structures. 


\section{ASSOCIATED CONTENT}

\section{S Supporting Information}

The Supporting Information is available free of charge at https://pubs.acs.org/doi/10.1021/acs.nanolett.9b04160.

Experimental methods, characterization of the cell lines, images used for analysis, control experiments, and additional examples (PDF)

\section{AUTHOR INFORMATION}

\section{Corresponding Author}

*E-mail: wegners@mpip-mainz.mpg.de.

\section{ORCID}

Seraphine V. Wegner: 0000-0002-9072-0858

\section{Funding}

This work was funded by the European Research Council ERC Starting Grant ARTIST (757593)

\section{Notes}

The authors declare no competing financial interest.

\section{ACKNOWLEDGMENTS}

We would like to thank Dr. Stefanie Möckel and Dr. Jesús Gil Pulido atthe IMB Flow Cytometry Core Facility (FCCF) for sorting our cell lines with flow cytometry. The pQE-80L iLID (C530M) (Addgene plasmid no. 60408) and pQE-80L MBPSspB Nano (Addgene no. 60409) plasmids were gifts from Brian Kuhlman. We thank Stefan Schuhmacher for the cover design.

\section{ABBREVIATIONS}

DLCA, diffusion-limited cluster aggregation; RLCA, reactionlimited cluster aggregation; PDGFR, platelet-derived growth factor receptor.

\section{REFERENCES}

(1) Liu, J. S.; Gartner, Z. J. Directing the assembly of spatially organized multicomponent tissues from the bottom-up. Trends Cell Biol. 2012, 22 (12), 683-691.

(2) Steinberg, M. S.; Poole, T. J. Strategies for specific form and pattern: adhesion-guided multicellular assembly. Philos. Trans. R. Soc., B 1981, 295 (1078), 451-460.

(3) Steinberg, M. S. Goal-Directedness in Embryonic Development. Integr. Biol. 1998, 1 (2), 49-59.

(4) Nichol, J. W.; Khademhosseini, A. Modular tissue engineering: engineering biological tissues from the bottom up. Soft Matter 2009, 5 (7), 1312-1319.

(5) Harrison, S. E.; Sozen, B.; Christodoulou, N.; Kyprianou, C.; Zernicka-Goetz, M. Assembly of embryonic and extraembryonic stem cells to mimic embryogenesis in vitro. Science 2017, 356 (6334), eaal1810.

(6) Rivron, N. C.; Frias-Aldeguer, J.; Vrij, E. J.; Boisset, J. C.; Korving, J.; Vivié, J.; Truckenmüller, R. K.; Van Oudenaarden, A.; Van Blitterswijk, C. A.; Geijsen, N. Blastocyst-like structures generated solely from stem cells. Nature 2018, 557 (7703), 106.

(7) Wan, A. C. A. Recapitulating Cell-Cell Interactions for Organoid Construction - Are Biomaterials Dispensable? Trends Biotechnol. 2016, 34 (9), 711-721.

(8) Toda, S.; Blauch, L. R.; Tang, S. K. Y. Y.; Morsut, L.; Lim, W. A. Programming self-organizing multicellular structures with synthetic cell-cell signaling. Science 2018, 361 (6398), 156-162.

(9) Merindol, R.; Walther, A. Materials learning from life: concepts for active, adaptive and autonomous molecular systems. Chem. Soc. Rev. 2017, 46 (8), 5588-5619.

(10) Valignat, M.-P.; Theodoly, O.; Crocker, J. C.; Russel, W. B.; Chaikin, P. M. Reversible self-assembly and directed assembly of
DNA-linked micrometer-sized colloids. Proc. Natl. Acad. Sci. U. S. A. 2005, 102 (12), 4225-4229.

(11) Li, F.; Josephson, D. P.; Stein, A. Colloidal Assembly: The Road from Particles to Colloidal Molecules and Crystals. Angew. Chem., Int. Ed. 2011, 50 (2), 360-388.

(12) Jungblut, S.; Joswig, J. O.; Eychmüller, A. Diffusion-Limited Cluster Aggregation: Impact of Rotational Diffusion. J. Phys. Chem. C 2019, 123 (1), 950-954.

(13) Lin, M. Y.; Lindsay, H. M.; Weitz, D. A.; Ball, R. C.; Klein, R.; Meakin, P. Universal diffusion-limited colloid aggregation. Phys. Rev. A: At., Mol., Opt. Phys. 1990, 41 (4), 2005.

(14) Tang, S.; Preece, J. M.; McFarlane, C. M.; Zhang, Z. Fractal Morphology and Breakage of DLCA and RLCA Aggregates. J. Colloid Interface Sci. 2000, 221 (1), 114-123.

(15) Selden, N. S.; Todhunter, M. E.; Jee, N. Y.; Liu, J. S.; Broaders, K. E.; Gartner, Z. J. Chemically Programmed Cell Adhesion with Membrane-Anchored Oligonucleotides. J. Am. Chem. Soc. 2012, 134 (2), 765-768.

(16) Sarkar, D.; Spencer, J. A.; Phillips, J. A.; Zhao, W.; Schafer, S.; Spelke, D. P.; Mortensen, L. J.; Ruiz, J. P.; Vemula, P. K.; Sridharan, R.; Kumar, S.; Karnik, R.; Lin, C. P.; Karp, J. M. Engineered cell homing. Blood 2011, 118 (25), e184-e191.

(17) Luo, W.; Pulsipher, A.; Dutta, D.; Lamb, B. M.; Yousaf, M. N. Remote Control of Tissue Interactions via Engineered Photoswitchable Cell Surfaces. Sci. Rep. 2015, 4, 6313.

(18) Shi, P.; Ju, E.; Yan, Z.; Gao, N.; Wang, J.; Hou, J.; Zhang, Y.; Ren, J.; Qu, X. Spatiotemporal control of cell-cell reversible interactions using molecular engineering. Nat. Commun. 2016, 7, 13088 .

(19) Shi, P.; Zhao, N.; Lai, J.; Coyne, J.; Gaddes, E. R.; Wang, Y. Polyvalent Display of Biomolecules on Live Cells. Angew. Chem., Int. Ed. 2018, 57 (23), 6800.

(20) Yüz, S. G.; Rasoulinejad, S.; Mueller, M.; Wegner, A. E.; Wegner, S. V. Blue Light Switchable Cell-Cell Interactions Provide Reversible and Spatiotemporal Control Towards Bottom-Up Tissue Engineering. Advanced Biosystems 2019, 3 (4), 1800310.

(21) Foty, R. A.; Steinberg, M. S. Differential adhesion in model systems. Wires. Dev. Biol.. 2013, 2 (5), 631-645.

(22) Duguay, D.; Foty, R. A.; Steinberg, M. S. Cadherin-mediated cell adhesion and tissue segregation: qualitative and quantitative determinants. Dev. Biol. 2003, 253 (2), 309-323.

(23) Katsamba, P.; Carroll, K.; Ahlsen, G.; Bahna, F.; Vendome, J.; Posy, S.; Rajebhosale, M.; Price, S.; Jessell, T. M.; Ben-Shaul, A.; Shapiro, L.; Honig, B. H. Linking molecular affinity and cellular specificity in cadherin-mediated adhesion. Proc. Natl. Acad. Sci. U. S. A. 2009, 106 (28), 11594-11599.

(24) Foty, R. A.; Steinberg, M. S. The differential adhesion hypothesis: a direct evaluation. Dev. Biol. 2005, 278 (1), 255-263.

(25) Foty, R. A.; Steinberg, M. S. Cadherin-mediated cell-cell adhesion and tissue segregation in relation to malignancy. Int. J. Dev. Biol. 2004, 48 (5-6), 397-409.

(26) Canty, L.; Zarour, E.; Kashkooli, L.; François, P.; Fagotto, F. Sorting at embryonic boundaries requires high heterotypic interfacial tension. Nat. Commun. 2017, 8 (1), 157.

(27) Han, K.; Go, D.; Tigges, T.; Rahimi, K.; Kuehne, A. J. C.; Walther, A. Social Self-Sorting of Colloidal Families in Co-Assembling Microgel Systems. Angew. Chem., Int. Ed. 2017, 56 (8), 2176-2182.

(28) Sentürk, O. I.; Chervyachkova, E.; Ji, Y.; Wegner, S. V. Independent Blue and Red Light Triggered Narcissistic Self-Sorting Self-Assembly of Colloidal Particles. Small 2019, 15, 1901801.

(29) Chervyachkova, E.; Wegner, S. V. Reversible Social Self-Sorting of Colloidal Cell-Mimics with Blue Light Switchable Proteins Elizaveta. ACS Synth. Biol. 2018, 7 (7), 1817-1824.

(30) Nieman, M. T.; Prudoff, R. S.; Johnson, K. R.; Wheelock, M. J. N-Cadherin Promotes Motility in Human Breast Cancer Cells Regardless of their E-Cadherin Expression. J. Cell Biol. 1999, 147 (3), 631-643.

(31) Guntas, G.; Hallett, R. a.; Zimmerman, S. P.; Williams, T.; Yumerefendi, H.; Bear, J. E.; Kuhlman, B. Engineering an improved 
light-induced dimer (iLID) for controlling the localization and activity of signaling proteins. Proc. Natl. Acad. Sci. U. S. A. 2015, 112 (1), $112-117$.

(32) Kawano, F.; Suzuki, H.; Furuya, A.; Sato, M. Engineered pairs of distinct photoswitches for optogenetic control of cellular proteins. Nat. Commun. 2015, 6, 6256.

(33) Bartelt, S. M.; Chervyachkova, E.; Steinkuhler, J.; Ricken, J.; Wieneke, R.; Tampe, R.; Dimova, R.; Wegner, S. V. Dynamic blue light-switchable protein patterns on giant unilamellar vesicles. Chem. Commun. 2018, 54 (8), 948-951.

(34) Aoki, K.; Kumagai, Y.; Sakurai, A.; Komatsu, N.; Fujita, Y.; Shionyu, C.; Matsuda, M. Stochastic ERK Activation Induced by Noise and Cell-to-Cell Propagation Regulates Cell Density-Dependent Proliferation. Mol. Cell 2013, 52 (4), 529-540.

(35) Melendez, J.; Patel, M.; Oakes, B. L.; Xu, P.; Morton, P.; Mcclean, M. N. Real-time optogenetic control of intracellular protein concentration in microbial cell cultures. Integr. Biol. 2014, 6, 366372.

(36) Lazzari, S.; Nicoud, L.; Jaquet, B.; Lattuada, M.; Morbidelli, M. Fractal-like structures in colloid science. Adv. Colloid Interface Sci. 2016, 235, 1-13.

(37) Meakin, P. Diffusion-controlled aggregation on two-dimensional square lattices: Results from a new cluster-cluster aggregation model. Phys. Rev. B: Condens. Matter Mater. Phys. 1984, 29, 29302941. 